\title{
Controversies in the anesthetic management of lumbar drains for endovascular aortic surgery
}

\author{
Sarah Marie Cardillo and Wendy K. Bernstein* \\ Department of Anesthesiology, University of Maryland School of Medicine, USA
}

\begin{abstract}
Lumbar drain insertion is an integral aspect of perioperative care for patients undergoing thoracoabodminal aortic aneurysm (TAA) repair. It has been shown to reduce incidence of paraplegia when both open and endovascular technique is used. Controversy exists in terms of optimal management of these catheters. This commentary aims to present data as well as experienced recommendation in their use.
\end{abstract}

Despite the increased use of endovascular techniques for thoracoabdominal aortic aneurysms (TAA), the risk of paraplegia after repair remains a significant concern for both the surgical and anesthesiology teams. Spinal cord ischemia or infarction with subsequent neurologic dysfunction occurs with an incidence of $4-13 \%$ after thoracic endovascular aortic repair (TEVAR) [1-3] Decreased spinal cord perfusion persists postoperatively when episodes of hypotension, hemorrhage and elevated cerebrospinal fluid (CSF) pressure occur, which further increases likelihood of paraplegia after TEVAR [4].

The highest risk factors for paraplegia after TEVAR are the extent of endovascular stent coverage and prior distal aortic repair [5]. Increasing spinal cord perfusion through augmentation of arterial pressure, lumbar CSF drainage and reattachment of segmental arteries has been shown to reduce the incidence of paraplegia. Spinal cord perfusion pressure is a function of lumbar CSF pressure subtracted from mean arterial pressure (MAP). Lumbar CSF drainage effectively improves spinal cord perfusion pressure via reduction of CSF pressure. Two separate metaanalyses including 3 randomized controlled trials have demonstrated the efficacy of controlled drainage of CSF for prevention of neurological injury [6,7].

The placement of lumbar CSF drains is relatively safe and recommended for TEVAR. However, contraindications to placement include pre-existing coagulopathy, elevated intracranial pressure, and frequently, emergent surgery [8]. The procedure itself carries potential side effects, including intracranial hemorrhage (ICH) and neuraxial hematoma. The American Society of Regional Anesthesia and Pain Medicine (ASRA) advises that instrumentation of the neuraxis be avoided in patients with preexisting coagulopathy, which may increase risk of bleeding with intrathecal catheter insertion. This risk is elevated with recent or concurrent administration of antiplatelet agents or anticoagulants. In addition, guidelines state that the time from the procedure and anticoagulation dosing should exceed 60 minutes and that the lowest dose of heparin be administered [9].

CSF drainage can be performed prior to the operation in high risk patients or after the procedure if symptoms of spinal cord ischemia occur. The timing of placement varies by center. Benefits of pre-induction placement include increased length of time prior to anticoagulation as well as knowledge of the patient's pain or paresthesis during placement, which could indicate potential nerve injury. In patients prescreened as high risk or difficult placement, it may be beneficial to place the lumbar drain well before the start of the case when alternative methods such as fluoroscopy can be utilized. Unfortunately, such management requires the patient to be hospitalized preoperatively which increases cost. Furthermore, prolonged catheter insertion can also place the patient at increased risk for infection [10].

The drain should be placed at the L3-4 or L4-5 level in either the seated or lateral decubitus position. Positioning is at the discretion of the anesthesiologist, as both positions offer advantages. The seated position allows for easier palpation of spinous processes through a midline insertion, which avoids the more lateral epidural plexus. In addition, the improved lumbar flexion allows potentially more space for needle insertion. Thus, the risk for bloody tap is decreased. On the other hand, the lateral decubitus position limits hydrostatic pressure of CSF and therefore the potential risk of over-draining CSF. The drain should be inserted $8-10 \mathrm{~cm}$, however depths as great as $30 \mathrm{~cm}$ have been reported [11]. Although deeper insertion minimizes the risk of inadvertent removal, it may also contribute to paresthesias and possible nerve root injuries [8].

Should a bloody tap be encountered during placement, the appropriate response remains unclear? Traumatic instrumentation of the neuraxis has been associated with up to $50 \%$ of neuraxial hematomas [8,9]. The occurrence of neuraxial hematoma is rare. In a case series of 162 patients undergoing full heparinization for extracorporeal circulation, there were no spinal hematomas [12]. Another retrospective study, however, reported two spinal hematomas in sixty five patients undergoing TAA repair [11]. At ours and other institutions, if a bloody tap is encountered, the practice is to discontinue

Correspondence to: Wendy K. Bernstein, Department of Anesthesiology, University of Maryland School of Medicine, S11 C 22 S Greene Street, Baltimore, Maryland 21201, USA, Tel: 410-328-6120; E-mail: WBERNSTEIN@anes.umm.edu

Received: May 08, 2015; Accepted: June 06, 2015; Published: June 10, 2015 
drainage and proceed with placement at another level. If bloody tap results a second time, case cancellation is considered after discussion with the operative team [13]. It has been suggested that the case be delayed up to 24 hours, however, based on ASRA guidelines this is only indicated for cases involving full heparinization for cardiopulmonary bypass, and otherwise there is no data supporting case cancellation. 8 9 Animal models have demonstrated a decreased risk for ischemic injury when goal CSF pressure is below $10 \mathrm{mmHg}$ [6]. Studies finding CSF drainage ineffective limited the total CSF drained to $50 \mathrm{~mL}$ as well as allowing the CSF pressure to elevate above $10 \mathrm{mmHg}$ [6]. Continuous monitoring intraoperatively with intermittent drainage allows awareness of sudden waveform disruption and possible drain occlusion. It also avoids high volume or rate drainage and potential intracranial hypotension. Protocols for CSF drainage from the lumbar drain range from 10 to $20 \mathrm{~mL} /$ hour depending on patient condition [8].

Management postoperatively requires vigilance in serial neurologic examination. In asymptomatic patients, management goals of CSF pressure should continue to be below $10 \mathrm{mmHg}$ with a rate limit of 10$15 \mathrm{~mL} / \mathrm{h}$. Patients with neurologic deficit may require drainage down to $5 \mathrm{mmHg}$ or up to $20 \mathrm{~mL} /$ hour, which has been shown to improve symptoms [8,13] At our institution, the catheter is opened to passive drainage for CSF pressures greater than $10 \mathrm{mmHg}$, and pressure is then reassessed after each $15 \mathrm{~mL}$ of CSF drained. Maximum CSF drainage rate is $20 \mathrm{~mL} /$ hour and up to $250 \mathrm{~mL} /$ day. If the pressure is persistently elevated after $150 \mathrm{~mL}$ are drained, the drain position is evaluated and the transducer re-zeroed. If the patient reveals any neurologic signs or symptoms, the catheter is drained to below $10 \mathrm{mmHg}$ coupled with blood pressure augmentation to a MAP $>90 \mathrm{mmHg}$.

Blood in the draining CSF is another issue that may be encountered. This may be indication of an intracranial bleed, the risk of which is associated with excessive CSF drainage and development of intracranial hypotension $[14,15]$. Bloody drainage has been shown to be a poor marker of an epidural cord hematoma [10]. Should a change in neurologic status accompany blood within the drain, recommended workup includes immediate imaging $[8,13]$, At our institution, brain imaging is additionally recommended for asymptomatic persistent bloody CSF drainage lasting more than 4 hours.

Based on current literature, drainage should be discontinued after 2-3 days if there is no sign of spinal cord ischemia. After this period, a fibrotic response will have occurred around the catheter, which seals the puncture site. Clamping the drain prior to removal may lead to greater chance of CSF leak secondary to elevated CSF pressure [13]. However, our institution recommends that the drain be clamped for 24 hours and the patient monitored for neurologic symptoms prior to removal. If a CSF leak occurs, conservative measures such as bed rest, limiting head of bed elevation and hydration are recommended [13].

Lumbar catheters are usually removed if there is no sign of neurologic dysfunction or spinal cord ischemia. Removal of the lumbar drain should always be performed by an experienced practitioner who is familiar with the catheters. Pulling the catheter out slowly and steadily will reduce the risk of catheter fracture. Positioning in the lateral decubitus position with hip and back flexion increases the space between the vertebrae and spinous processes. This reduces the force required for removal, thus reducing the risk of fracturing the catheter [8]. If catheter breakage occurs, it is essential to perform a CT scan of the spine to determine the track and position of the retained fragment. The decision to remove the retained portion should be made on a case by case basis [16].
In our institution, it is also recommended that a full coagulation profile be performed prior to lumbar drain removal. For patients on a heparin infusion, it should be discontinued 2-4 hours prior to removal and not restarted for a full 12 hours afterward. It is prudent to wait 6-8 hours to start prophylactic once daily dosing and the catheter can be removed 12 hours after the last dose of once daily low molecular weight heparin. Twice daily dosing of low molecular weight heparin (LMWH) is associated with increased risk of spinal hematoma. The first dose of LMWH should be administered no earlier than 24 hours postoperatively. Indwelling catheters should be removed prior to initiation of LMWH thromboprophylaxis. Adequate surgical hemostasis and reversal of coagulopathy should be assured [9].

The optimal management of lumbar drain catheters for TEVAR lacks consensus and requires further evaluation. Given the number of studies revealing benefit and rare incidence of complications, the procedure will continue to be an integral aspect of perioperative care of these patients. Vigilance is required to evaluate the patient not only for neurologic sequelae of their operation, but for potentially catastrophic consequences of the indwelling drain. Through the use of education and awareness at all levels of care, reduction in paraplegia can be safely achieved.

\section{References}

1. Greenberg RK, Lu Q, Roselli EE, Svensson LG, Moon MC, et al. (2008) Contemporary analysis of descending thoracic and thoracoabdominal aneurysm repair: a comparison of endovascular and open techniques. Circulation 118: 808-817. [Crossref]

2. Xenos ES, Minion DJ, Davenport DL, Hamdallah O, Abedi NN, et al. (2009) Endovascular versus open repair for descending thoracic aortic rupture: institutional experience and meta-analysis. Eur J Cardiothorac Surg 35: 282-286. [Crossref]

3. Jonker FH, Trimarchi S, Verhagen HJ, Moll FL, Sumpio BE, et al. (2010) Metaanalysis of open versus endovascular repair for ruptured descending thoracic aortic aneurysm. J Vasc Surg 51: 1026-1032, 1032. [Crossref]

4. Safi HJ, Miller CC, Azizzadeh A, Iliopoulos DC (1997) Observations on delayed neurologic deficit after thoracoabdominal aortic aneurysm repair. J Vasc Surg 26: 616622. [Crossref]

5. Messé SR, Bavaria JE, Mullen M, Cheung AT, Davis R, et al. (2008) Neurologic outcomes from high risk descending thoracic and thoracoabdominal aortic operations in the era of endovascular repair. Neurocrit Care 9: 344-351. [Crossref]

6. Cinà CS, Abouzahr L, Arena GO, Laganà A, Devereaux PJ, et al. (2004) Cerebrospinal fluid drainage to prevent paraplegia during thoracic and thoracoabdominal aortic aneurysm surgery: a systematic review and meta-analysis. J Vasc Surg 40: 36-44. [Crossref]

7. Khan SN, Stansby G (2004) Cerebrospinal fluid drainage for thoracic and thoracoabdominal aortic aneurysm surgery. Cochrane Database Syst Rev 1: CD003635. [Crossref]

8. Fedorow CA, Moon MC, Mutch WA, Grocott HP (2010) Lumbar cerebrospinal fluid drainage for thoracoabdominal aortic surgery: rationale and practical considerations for management. Anesth Analg 111: 46-58. [Crossref]

9. Horlocker TT, Wedel DJ, Rowlingson JC, Enneking FK, Kopp SL, et al. (2010) Regional anesthesia in the patient receiving antithrombotic or thrombolytic therapy: American Society of Regional Anesthesia and Pain Mdicine Evidence-Based Guidelines (third edition). Reg Anesth Pain Med 35: 64-101. [Crossref]

10. Weigang E, Hartert M, Siegenthaler MP, Beckmann NA, Sircar R, et al. (2006) Perioperative management to improve neurologic outcome in thoracic or thoracoabdominal aortic stent-grafting. Ann Thorac Surg 82: 1679-1687. [Crossref]

11. Weaver K, Wiseman D, Farber M, Ewend M, Marston W, et al. (2001) Clinical Research Studies: Complications of lumbar drainage after thoracoabdominal aortic aneurysm repair. Journal of Vascular Surgery 34: 623-627.

12. Cheung AT, Pochettino A, Guvakov DV, Weiss SJ, Shanmugan S, et al. (2003) Safety of lumbar drains in thoracic aortic operations performed with extracorporeal circulation. Ann Thorac Surg 76: 1190-1196. [Crossref] 
13. Estrera AL, Sheinbaum R, Miller CC, Azizzadeh A, Walkes JC, et al. (2009) Cerebrospinal Fluid Drainage During Thoracic Aortic Repair: Safety and Current Management. The Annals of Thoracic Surgery 88: 9-15. [Crossref]

14. Vaughn SB, LeMaire SA, Collard CD (2011) Case scenario: anesthetic considerations for thoracoabdominal aortic aneurysm repair. Anesthesiology 115: 1093-1102. [Crossref]
15. Sinha AC, Cheung AT (2010) Spinal cord protection and thoracic aortic surgery. Curr Opin Anaesthesiol 23: 95-102. [Crossref]

16. Olivar H, Bramhall JS, Rozet I, Vavilala MS, Souter MJ, et al. (2007) Subarachnoid lumbar drains: a case series of fractured catheters and a near miss. Can J Anaesth 54: 829-834. [Crossref]

Copyright: (C2015 Cardillo SM. This is an open-access article distributed under the terms of the Creative Commons Attribution License, which permits unrestricted use, distribution, and reproduction in any medium, provided the original author and source are credited. 